\title{
Paralelização Híbrida e em Múltiplos Níveis de um Algoritmo de Contabilização de Frequências de $K$-mer
}

\author{
Fabrício Vilasbôas ${ }^{1}$, Micaella Coelho ${ }^{1}$, \\ Carla Osthoff $^{1}$, Kary Ocaña ${ }^{1}$, Ana Tereza Vasconcelos ${ }^{1}$ \\ ${ }^{1}$ Laboratório Nacional de Computação Científica \\ \{fabricio, micaella, osthoff, karyann, atrv\}@lncc.br
}

\begin{abstract}
Resumo. Este trabalho apresenta um estudo sobre o ganho de desempenho gerado com o aumento do nível de paralelismo de execução de um algoritmo de contabilização de k-mers de dados de amostras genômicas e metagenômicas. $O$ algoritmo foi originalmente desenvolvido para permitir a execução em paralelo da contabilização de k-mers em uma arquitetura manycores de uma GPU. Neste trabalho apresentamos o ganho de desempenho ao implementarmos paralelismo para a execução paralela de módulos do algoritmo em múltiplos núcleos de uma CPU, em múltiplas GPU's e em múltiplos nós de processamento de uma cluster híbrida.
\end{abstract}

\section{Introdução}

Com o surgimento da tecnologia NGS (Next Generation Sequencing) muitos foram os avanços na área da pesquisa genômica. As técnicas de sequenciamentos mais avançados geravam $84 \mathrm{MB}$ de dados por rodada e hoje, com o NGS, são gerados terabytes de dados por rodada. Os avanços na tecnologia de sequenciamento aumentaram a necessidade do desenvolvimento de técnicas para permitir a análise dos dados de forma mais rápida e precisa. Neste trabalho apresentamos otimizações de desempenho de um algoritmo de contabilização de $k$-mers para dados de amostras genômicas e metagenômicas, chamado de "Contabilizador da Frequência de Repetição de k-mers"ou CFRK.

O algoritmo CFRK [Vilasboas et al. 2015] foi desenvolvido para efetuar contabilização da frequência de repetição de k-mers de bases de dados de amostras genômicas em ambientes de GPU's. O termo k-mer é utilizado como referência a todas as possíveis combinações de comprimento $k$ que estão contidas em uma sequência de dados arbitrária. Essa técnica está contida na interseção entre a mineração de dados e a análise combinatória. As aplicações dos $k$-mers são inúmeras. K-mers podem ser utilizados para montagem [Garg et al. 2013] de amostras de DNA, RNA ou proteínas, para alinhamento de sequências [Church et al. 2011], ou para análise qualitativa de um sequenciamento [Plaza Onate et al. 2015]. Também podem ser utilizados como entrada para algum método de classificação ou agrupamento [Fiannaca et al. 2015]. Os valores de $k$ utilizados variam de acordo com a análise feita.

A primeira versão do CFRK apresentou um ganho de aproximadamente 6 vezes em relação ao Jellyfish, considerado o estado da arte para contabilização de $k$-mer, para valores de $k$ menores que 5 , porém tinha a limitação de processar apenas arquivos de tamanho menor ou igual à memória principal da GPU. A segunda versão do CFRK 
[Vilasboas et al. 2016] implementou uma rotina para dividir os dados de entrada e possibilitou a execução de arquivos de tamanho maior do que a memória principal da GPU. Neste trabalho apresentamos a terceira versão do CFRK, que introduziu mais três níveis de paralelismo: um primeiro nível para permitir o processamento de parte do algorítimo em múltiplos núcleos do módulo $C P U$, um segundo nível para permitir o processamento de parte do algoritmo em múltiplas GPU's e um terceiro nível para permitir a execução de múltiplas instâncias do algoritmo CFRK em nós de processamento de uma cluster.

Este trabalho analisa o ganho de desempenho do CFRK para cada otimização implementada e o ganho de desempenho total do CFRK com todas as otimizações. Demonstramos que a adição de mais 3 níveis de paralelismo aumenta significativamente o desempenho do algoritmo.

Este trabalho está dividido da seguinte forma: Seção 2 apresenta os trabalhos relacionados; Seção 3 apresenta a descrição do algoritmo CFRK e das otimizações desenvolvidas. Seção 4 apresenta a análise de desempenho. Seção 5 apresenta a descrição do MCFRK, resultados e análises. Seção 6 apresenta as conclusões e os trabalhos futuros.

\section{Trabalhos Relacionados}

O Jellyfish [Marçais and Kingsford 2011] é um algoritmo desenvolvido para processamento em memória compartilhada e é considerado o estado da arte entre os algoritmos de contabilização da frequência de repetição de $k$-mers. Ele utiliza várias estruturas lock-free, que são estruturas que permitem operações atômicas sem o bloqueio da memória e, por isso, não degradam o desempenho em ambientes multiprocessáveis. O Jellyfish apresenta bom desempenho para a contabilização de valores de $10 \leq k \leq 32$ enquanto que o CFRK possui melhor desempenho para valores de $k \leq 9$. Por outro lado, o Jellyfish foi projetado para processar amostras de genoma, pois ele considera todos os reads ${ }^{1}$ como sendo pertencente a um mesmo organismo. O CFRK foi projetado para permitir a análise de amostras de genomas e de metagenomas, pois considera que cada read pode pertencer a um organismo distinto. Isto possibilita uma análise conclusiva para grande classe de pesquisas em bioinformática, conforme o trabalho [Edwards et al. 2012].

O Gerbil [Erbert et al. 2017] é um algoritmo para contabilização da frequência de repetição de k-mers com suporte a múltiplas GPU's e que faz uso do disco para melhorar a eficiência em relação à memória. Sua execução consiste de duas fases: distribuição e contabilização. Este algoritmo é projetado para o processamento de genomas. Isto permite que o Gerbil efetue menos transferência de dados entre a GPU e o host e apresente um bom desempenho para valores de $k \leq 32$. Porém, assim como o Jellyfish, o Gerbil não pode ser utilizado para pesquisas que necessitam gerar um vetor de frequência para cada read.

\section{Descrição do algoritmo}

CFRK é acrônimo para "Contabilizador da Frequência de Repetição de k-mers", foi desenvolvido para GPU's da NVidia, utilizando a linguagem CUDA e os drivers disponibilizados pelo fabricante.

Para o desenvolvimento do CFRK utilizamos o conceito de programação modular, onde cada módulo é responsável por uma atividade específica na execução do algoritmo.

\footnotetext{
${ }^{1}$ Fragmentos de material genético resultantes do sequenciamento
} 
O CFRK é composto por dois módulos principais: o módulo que contém as funções que são executadas pela $C P U$, chamado módulo $C P U$, e o módulo que contém as funções que são executadas pela $G P U$, chamado módulo GPU. O módulo $C P U$ é composto por dois submódulos: o main e o kmer_main. O submódulo main é responsável pela leitura do arquivo de entrada, pela preparação dos chunks para serem enviados as GPU's e pela gerência da execução dos outros submódulos. O módulo kmer_main é responsável pela preparação da GPU para a execução e possui as rotinas de gerenciamento e transferência de memória e chamadas aos kernels. O módulo GPU é composto pelo submódulo kmer_kernel, que contém os kernels executadas na GPU.

\subsection{Descrição das funções do módulo $C P U$}

Nesta seção descreveremos o funcionamento dos dois submódulos contidos no módulo CPU, o submódulo main e o submódulo kmer_main.

\subsubsection{Submódulo main}

$\mathrm{O}$ arquivo resultante do sequenciamento de uma amostra genômica ou metagenômica contém um grande número de fragmentos, denominadas reads. Cada read possui uma sequência de nucleotídeos que são representados pelos caracteres $A, C, G$, $\mathrm{T}$ ou $\mathrm{N}$. O caractere $\mathrm{N}$ é observado no read quando o sequenciador não reconhece com precisão o nucleotídeo pertencente àquela posição. Quando o arquivo é lido, é feita uma codificação para transformar os caracteres $\mathrm{A}, \mathrm{C}, \mathrm{G}, \mathrm{T}$ ou $\mathrm{N}$ para os valores numéricos 0 , 1, 2, 3 ou -1, respectivamente. Os reads são dispostos em sequência formando uma estrutura de array unidimensional contendo todos os nucleotídeos de todos os reads, chamado vetor de reads. Para identificar o final de cada read, é utilizado o valor -1 . O valor -1 é utilizado para representar tipos de dados inválidos, ou seja, o k-mer que contém o valor -1 não deverá ser processado. Os arquivos gerados pelos sequenciadores de tecnologia $N G S$ podem conter uma grande quantidade de informação e a memória global da $G P U$ pode não ser suficiente para armazenar toda essa informação. Por isto o vetor de reads é subdividido e armazenado em estruturas chamadas chunks. Cada chunk possui uma quantidade pré-definida de reads, bem como as informações da posição inicial e o tamanho de cada read. Posteriormente, os chunks são enfileirados e serão enviados um a um para o processamento na GPU. Com isso pode-se ajustar a quantidade de dados que será enviada para o processamento na $G P U$ e, assim, tem-se o controle da quantidade total de memória utilizada para o processamento. O usuário tem liberdade para definir a quantidade de reads por chunk, sendo que a quantidade máxima varia de acordo com a disponibilidade da memória da placa que está em utilização. Esta função demanda um grande tempo de processamento devido a manipulação de memória. Esta função pertence ao módulo main, no qual estão todas as funções que são executadas exclusivamente pela $C P U$.

\subsubsection{Submódulo $k m e r \_m a i n$}

Os chunks são enviados para o submódulo kmer_main através de uma estrutura que contém as sequências a serem processadas o número de sequências, o tamanho de cada sequência e a posição inicial de cada sequência. É neste módulo de que se encontram todas as funções de gerenciamento de memória, funções para alocação e desalocação 
dos vetores, funções de transferência de memória entre host e device e as chamadas aos kernels. Este módulo foi alvo do estudo publicado em [Vilasboas et al. 2016].

\subsubsection{Otimização no módulo $C P U$}

Foi feito um estudo do comportamento das funções executadas pela $C P U$ durante a execução do CFRK. Foi utilizada a ferramenta VTune disponibilizada pela Intel em seu pacote Intel Parallel Studio. Observamos nestes resultados que a função de leitura de arquivo e a função responsável pela seleção dos chunks compunham os hotspots. O VTune nos permitiu visualizar as instruções que mais demandaram processamento dentro destes hotspots e com base nestas informações foi selecionado um laço de repetição para a paralelização via diretivas do OpenMP.

O laço de repetição selecionado foi o que mais demandou processamento na função de seleção dos chunks. A operação realizada por este laço de repetição consiste em uma cópia de memória entre dados lidos do arquivo de entrada e a estrutura que armazenará os chunks para o processamento. A diretiva inserida foi a \#pragma omp parallel for. Essa diretiva cria uma região paralela na qual as iterações do laço de repetição são divididas para serem executadas em paralelo pelos núcleos da $C P U$.

\subsection{Descrição das funções do módulo $G P U$}

O processo de contabilização da frequência de repetição de $k$-mers é dividido em duas fases. A primeira fase é a conversão dos valores da base 4 para a base 10 e a segunda fase é a contabilização da frequência de repetição de $k$-mers.

A primeira fase do processamento consiste em converter os valores numéricos dos $k$-mers, que estão na base 4 , para a base 10. Essa conversão é feita para facilitar o mapeamento entre o valor da combinação do $k$-mer e a posição do contador no vetor de frequência referente àquela combinação, dado que o valor do k-mer na base 10 será exatamente o valor da posição a qual deverá ser feita a contabilização da repetição. $\mathrm{O}$ resultado desta conversão é armazenado em um vetor denominado vetor de conversão.

$\mathrm{Na}$ segunda fase é calculada da frequência de repetição de cada $k$-mer. É alocado um vetor chamado vetor de frequência. Este vetor irá armazenar o resultado do cálculo da frequência de repetição. Para cada read no vetor de reads será necessário alocar um vetor de frequência e cada posição do vetor de frequência será um contador correspondente ao valor do k-mer no vetor de conversão. Então o vetor de conversão é percorrido e é realizada a operação +1 no vetor de frequência na posição correspondente ao valor do $k$-mer.

\subsubsection{Otimização no módulo $G P U$}

A GPU foi utilizada para acelerar o processamento da conversão dos valores dos $k$-mers da base 4 para a base 10 e também a contabilização da frequência de repetição dos k-mers. Essas funções foram selecionadas para este tipo de abordagem pelo fato de se enquadrarem no paradigma Single Instruction Multiple Data (SIMD), ou seja, vários dados sendo operados por uma instrução. Estas otimizações estão descritas no trabalho [Vilasboas et al. 2016]. 
Para este trabalho foi feita a alteração do tipo do dado utilizado no vetor de reads. Até a segunda versão do CFRK o vetor de reads era armazenado como int (inteiro), que ocupa 4 bytes na memória. A proposta foi alterar de int para o tipo char (caractere), que ocupa 1 byte na memória. Essa alteração foi possível porque a linguagem $C U D A$ é uma extensão da linguagem $C$ e esta usa o tipo do dado apenas como referência ao espaço de alocação em memória permitindo fazer cálculos com variáveis de qualquer tipo primitivo.

Essa alteração foi feita no kernel ComputeIndex do submódulo kmer_kernel que pertence ao módulo GPU.

\subsection{Suporte a execução em múltiplas GPU's}

De forma a possibilitar que a execução do CFRK em múltiplas GPU's, foi desenvolvida uma função no submódulo main que reconhece o número de GPU's disponíveis através da função cudaGetDeviceCount da biblioteca padrão do CUDA e utiliza a biblioteca pthreds para criar threads do módulo kmer_main para serem executadas em paralelo em cada GPU. A função desenvolvida, após gerar as threads, irá designar um grupo de chunks para serem processados em cada thread e a seguir enviar as threads para serem executadas em cada $G P U$. Após a execução os vetores de frequência gerados por cada GPU são coletados pelo módulo main e armazenados no arquivo de saída e as threads são destruídas.

\section{Resultados e análises}

Nesta seção apresentaremos os resultados referentes as otimizações implementadas nos dois módulos principais e seus respectivos resultados.

Para os experimentos foram utilizando os nós computacionais GPU's do supercomputador SDumont, onde cada nó possui a configuração: 2 x CPU Intel Xeon E52695v2 Ivy Bridge, 2,4GHZ, 24 núcleos (12 por $C P U$ ), totalizando de 1.296 núcleos, 64GB DDR3 RAM, 2 x Nvidia K40 (dispositivo GPU), sistema operacional Red Hat Enterprise Linux Server release 6.4, kernel versão 2.6.32-504.12.2.el6.x86_64, CUDA 8.0, Intel Parallel Studio XE 2016, compilador icpc com flags as flags -qopenmp e -Ofast, VTune e OpenMP 4.5. Este supercomputador está alocado no Laboratório Nacional de Computação Científica (LNCC) e para este trabalho foram utilizados os nós B715 com placas aceleradoras GPU Nvidia K40.

$\mathrm{O}$ arquivo de entrada utilizado foi uma amostra real de metagenoma com 8.7GB de dados cuja identificação no banco de dados SRA do NCBI (National Center for Biotechnology Information) é SRX2021688. Este arquivo foi escolhido por representar um típico arquivo de tamanho médio utilizado para pesquisas de metagenoma.

\subsection{Resultado das otimizações no módulo $C P U$}

Utilizamos o VTune para gerar o perfil de execução do CFRK paralelizado com a diretiva OpenMP. Dado que o valor de $k$ não é utilizado pelas rotinas dos submódulos da $C P U$, os resultados apresentados são relativos a um valor fixo de $k$. Escolhemos o valor $k=4$ que é utilizado para análise de genoma conforme o trabalho [Edwards et al. 2012]. Denominamos a versão do CFRK que possui os submódulos do módulo $C P U$ paralelizados através do OpenMP de CFRK_OMP. A Tabela 1 apresenta na primeira coluna o nome das funções das duas versões do algoritmo CFRK sendo a primeira linha referente 
a versão sem a diretiva $O p e n M P$, CFRK, e a segunda linha referente a versão com a diretiva OpenMP, CFRK_OMP. A segunda coluna apresenta o tempo de execução em segundos de cada uma das funções. A terceira coluna apresenta o ganho em vezes da função com a diretiva OpenMP em relação a mesma função sem a diretiva. Nossos expe-

\begin{tabular}{l|c|c} 
Função & Tempo (segundos) & Ganho (vezes) \\
\hline SelectChunk (CFRK) & 10.427 & - \\
SelectChunk (CFRK_OMP) & 0.303 & 34.41
\end{tabular}

Tabela 1: Resultado obtido através do VTune para o CFRK e CFRK_OMP

rimentos demonstram que a paralelização ao nível dos múltiplos núcleos da $C P U$ através da inserção de diretivas do OpenMP em um laço de repetição da função de cópia de dados do arquivo de entrada para uma estrutura de processamento gerou um ganho de 34 vezes para 12 núcleos em relação a execução com apenas um núcleo. Este ganho mostra que o ambiente multicore, além de aumentar a taxa de execução em paralela do algoritmo também ajuda a diminuir o tempo de acesso aos dados na memória cache, gerando um ganho "supralinear"para a função SelectChunk.

\subsection{Resultado das otimizações no módulo GPU}

O submódulo kmer_kernel, que executa na GPU, possui o kernel ComputeIndex que executa uma conversão dos valores dos $k$-mers da base 4 para a base 10 antes de realizar a contabilização da frequência de repetição dos $k$-mers. De forma a diminuir o espaço ocupado pelos dados na cache da GPU, realizamos a alteração do tipo do dado utilizado no vetor de reads. Originalmente este vetor era armazenado como int (inteiro), ocupando 4 bytes por elemento na memória. Este foi alterado para o tipo char (caractere), que ocupa 1 byte por elemento na memória. Essa alteração foi possível porque a linguagem CUDA é uma extensão da linguagem $C$ e esta usa o tipo do dado apenas como referência ao espaço de alocação em memória permitindo fazer cálculos com variáveis de qualquer tipo primitivo.

Os resultados apresentados nesta seção são da execução do kernel ComputeIndex e foram obtidos através do nvprof. As Tabelas 2, 3, 4 e 5 apresentam os resultados da execução do kernel ComputeIndex gerados pelo perfilador nvprof para os valores de $k=$ $\{2,3,4\}$ respectivamente. A primeira coluna apresenta o nome das versões do CFRK onde CFRK_INT se refere a versão original com o vetor de reads armazenado como inteiro e CFRK_CHAR se refere a nova versão com o vetor de reads armazenado como caractere. A segunda coluna apresenta a Ocupação que é a relação entre a média de warps ativos por ciclo e a quantidade máxima de warps suportada pela placa. A terceira coluna apresenta o IPC (Instructions per cycle) que é a quantidade de operações executadas por ciclo. A quarta coluna apresenta o valor médio de acessos repetidos à memória devido ao cache miss. A quinta coluna apresenta as Instruções por warp que é a média de instruções executadas por warp. A sexta coluna apresenta Warps por ciclo que é a quantidade média de warps ativos por ciclo. Ao observar as Tabelas 2, 3, 4 e 5 notamos uma grande diminuição da taxa de Cache miss e um consequente aumento do respectivo IPC da versão anterior, CFRK_INT, em relação a versão atual, CFRK_CHAR. Note que na versão CFRK_INT o valor de cache miss aumenta com proporção média de $3 \times 10^{-3}$ e a versão CFRK_CHAR aumenta com proporção média de $3 \times 10^{-4}$. Por consequência deste fato 


\begin{tabular}{c|ccccc} 
Versão & Ocupação & IPC & Cache miss & Inst. por Warp & Warps por ciclo \\
\hline CFRK_INT & $87.64 \%$ & 2.80 & 0.00599 & 82.92 & 7.11 \\
CFRK_CHAR & $92.26 \%$ & 4.07 & 0.00122 & 202.89 & 15.79
\end{tabular}

Tabela 2: Dados do perfilador para $k=2$

\begin{tabular}{c|ccccc} 
Versão & Ocupação & IPC & Cache miss & Inst. por Warp & Warps por ciclo \\
\hline CFRK_INT & $89.30 \%$ & 2.80 & 0.00956 & 103.90 & 6.68 \\
CFRK_CHAR & $93.96 \%$ & 4.07 & 0.00175 & 282.84 & 17.72
\end{tabular}

Tabela 3: Dados do perfilador para $k=3$

\begin{tabular}{c|ccccc} 
Versão & Ocupação & IPC & Cache miss & Inst. por Warp & Warps por ciclo \\
\hline CFRK_INT & $91.06 \%$ & 2.77 & 0.01193 & 124.87 & 6.27 \\
CFRK_CHAR & $94.87 \%$ & 3.94 & 0.00205 & 362.78 & 18.03
\end{tabular}

Tabela 4: Dados do perfilador para $k=4$

\begin{tabular}{c|ccccc} 
Versão & Ocupação & IPC & Cache miss & Inst. por Warp & Warps por ciclo \\
\hline CFRK_INT & $91.58 \%$ & 2.80 & 0.01362 & 145.85 & 7.55 \\
CFRK_CHAR & $94.55 \%$ & 4.03 & 0.00224 & 442.73 & 17.93
\end{tabular}

Tabela 5: Dados do perfilador para $k=5$

todas as outras taxas aumentam. A Ocupação aumentou $4 \%$ em média, o valor do IPC teve um aumento de aproximadamente 1.4 vezes, o número de Instruções por Warp teve um aumento de 3 vezes em média, o número de Warps por ciclo teve um aumento de 2.5 vezes em média.

Isto se deve pela diminuição da ocupação da memória. Ao trocar o tipo de dado inteiro, 4 bytes, para caractere, 1 byte, foi possível alocar 4 vezes mais dados nas memórias cache da GPU. Isso fez com que as requisições à memória global diminuíssem, aumentando a performance deste kernel.

\subsection{Avaliação de desempenho do CFRK com 3 níveis de paralelismo}

A Figura 1 apresenta o tempo de execução do CFRK da versão sem as otimizações apresentadas neste trabalho, identificada no gráfico como orig, em relação a versão com todas as otimizações apresentadas neste trabalho, identificada no gráfico como CFRK_OMP_MG. O eixo $x$ apresenta o tempo de execução em segundos e o eixo $y$ apresenta os valores de $k$. A Tabela 6 apresenta a redução do tempo obtido pelos aprimoramentos feitos na versão inicial do CFRK. A primeira linha apresenta os valores de $k$, a segunda linha apresenta o tempo em segundos e a terceira linha apresenta o ganho em porcentagem. Observando a Figura 1 e a Tabela 6 vemos que o tempo de processamento foi reduzido em mais de 80 segundos em todos os casos. A maior redução no tempo foi de $39.92 \%$ para $\mathrm{k}=2$, que executa menos operações de transferência de dados entre a $G P U$ e o host. Conforme análise apresentada em trabalhos anteriores [Vilasboas et al. 2016], a medida que o valor de $k$ aumenta, a complexidade do tempo de execução do kernel de contabilização de frequência de $k$-mer aumenta. Por outro lado, a medida que aumenta o valor de $k$ aumenta o tamanho do vetor de contabilização de $k$-mers e consequentemente 


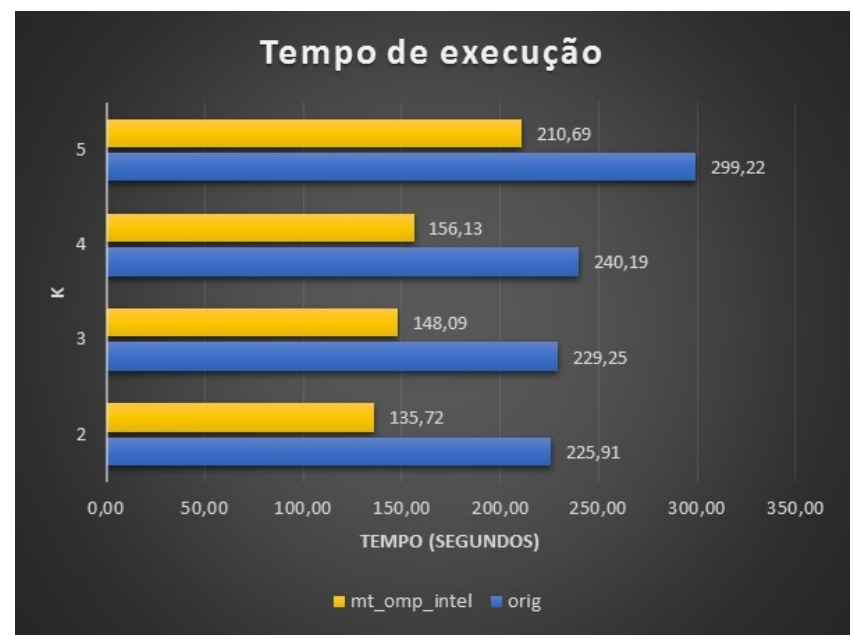

Figura 1: Tempo de execução do CFRK e do CFRK_OMP_MG

\begin{tabular}{l|llll}
$\mathrm{k}$ & 2 & 3 & 4 & 5 \\
\hline Tempo (segundos) & 90.19 & 81.16 & 84.06 & 88.53 \\
Ganho (\%) & 39.92 & 35.40 & 34.99 & 29.58
\end{tabular}

\section{Tabela 6: Tempo de processamento reduzido entre o CFRK_OMP_MG em relação ao CFRK}

o tempo com a transferência de dados entre a $G P U$ e o host, justificando a diminuição do ganho.

\section{MCFRK}

O MCFRK [Coelho et al. 2016] foi desenvolvido para possibilitar a execução de forma eficiente do CFRK com arquivos que possuem tamanho maior ou igual a memoria principal da máquina em execução. MCFRK é o acrônimo de MPI - Contabilizador da Frequência de Repetição de K-mers. Este algoritmo é uma extensão do CFRK para permitir a execução em ambientes de memoria distribuída, ou cluster, composta por diversos nós computacionais com placas aceleradoras GPU's. Para isso, foi utilizado o padrão de troca de mensagens MPI. A versão do MCFRK apresentada nesta seção integra todas as otimizações relatadas nas seções anteriores.

O MCFRK decompõe o arquivo original em arquivos menores para serem processados nos múltiplos nós de processamento da cluster. Foram adicionadas ao submódulo main do módulo CPU funções da biblioteca MPI para enviar, criar e gerenciar a execução de processos CFRK nos nós de processamento. A execução do MCFRK é dividida em duas fases: o pré-processamento e o processamento. Na fase de pré-processamento é realizada a divisão do arquivo. Esta divisão é realizada através de uma aplicação desenvolvida na linguagem $\mathrm{C}$, chamada de split, que realiza o particionamento do arquivo em arquivos menores com o mesmo número de reads. Na fase de processamento e após a inicialização dos processos $M P I$, é fornecido a localização dos respectivos arquivos de entrada e de saída. Cada processo MPI realiza a execução de uma instância do CFRK e ao final do processamento os resultados do arquivo de saída são armazenados em disco. 


\subsection{Resultados e análise}

Primeiramente vamos apresentar o resultado de cada uma das otimizações separadamente e posteriormente apresentar uma análise mais detalhada do MCFRK.

Para os experimentos apresentados na Tabela 7 foram utilizados 8 nós do supercomputador SDumont, onde cada nó possui as configurações apresentadas na Seção 4. $\mathrm{O}$ arquivo de entrada utilizado foi uma amostra real de metagenoma com 8.7GB de dados cuja identificação no banco de dados SRA do NCBI (National Center for Biotechnology Information) é SRX2021688. A Tabela 7 apresenta o tempo de execução e o ganho para cada otimização aplicada ao MCFRK. A primeira coluna apresenta a descrição da otimização; a segunda coluna apresenta os tempos de execução de cada versão com 1 processo MPI; a terceira coluna apresenta os tempos de execução de cada versão com 8 processos $M P I$ sem a contabilização da operação de split; a quarta coluna apresenta os tempos de execução de cada versão com 8 processos $M P I$ com a contabilização da operação de split; a quinta coluna apresenta o ganho de performance de cada versão sem a contabilização da operação de split; a sexta coluna apresenta o ganho de performance de cada versão sem a contabilização da operação de split.

\begin{tabular}{l|lllll} 
Versão & 1 processo & 8 processos sem split & 8 processos com split & Ganho sem split & Ganho com split \\
\hline Sem otimização & 160.39 & 20.4 & 70.545 & 7.86 & 2.27 \\
Otimização módulo CPU & 151.18 & 19.22 & 69.365 & 7.87 & 2.18 \\
Otimização módulo GPU & 155.97 & 19.99 & 70.135 & 7.80 & 2.22 \\
Todas as otimizações & 144.64 & 18.07 & 68.215 & 8,00 & 2.12
\end{tabular}

Tabela 7: Tempo de execução e ganho de cada otimização aplicada ao MCFRK

Para os experimentos apresentados nas Figuras $2 \mathrm{a}$ e $2 \mathrm{~b}$ foram utilizados 14 nós do supercomputador SDumont, onde cada nó possui as configurações apresentadas na Seção 4. O arquivo de entrada utilizado foi uma amostra real de metagenoma com 15GB de dados cuja identificação no banco de dados SRA do NCBI (National Center for Biotechnology Information) é SRX1142487.

A Figura 2a apresenta o ganho obtido pela execução do MCFRK com múltiplos processos em relação a execução dele próprio com um processo sem incluir o tempo da fase de pré-processamento. A linha em azul escuro apresenta o ganho com 2 processos, a linha em laranja apresenta o ganho com 4 processos, a linha em amarelo apresenta o ganho com 6 processos, a linha em verde apresenta o ganho com 8 processos, a linha em vinho apresenta o ganho com 10 processos, a linha em azul claro apresenta o ganho com 12 processos, a linha em verde escuro apresenta o ganho com 14 processos. A Figura $2 \mathrm{~b}$ apresenta o ganho obtido pela execução do MCFRK com múltiplos processos em relação a execução dele próprio com um processo incluindo o tempo de pré-processamento. A linha em azul escuro apresenta o ganho com 2 processos, a linha em laranja apresenta o ganho com 4 processos, a linha em amarelo apresenta o ganho com 6 processos, a linha em verde apresenta o ganho com 8 processos, a linha em vinho apresenta o ganho com 10 processos, a linha em azul claro apresenta o ganho com 12 processos, a linha em verde escuro apresenta o ganho com 14 processos. A diferença entre os ganhos de desempenho nas Figuras 2a e 2b, sem e com a operação split, mostram o impacto no desempenho do sistema causado pelas operações em disco. Conforme explicado anteriormente, a operação split é executada na fase de pré-processamento onde é feito o particionamento do arquivo 


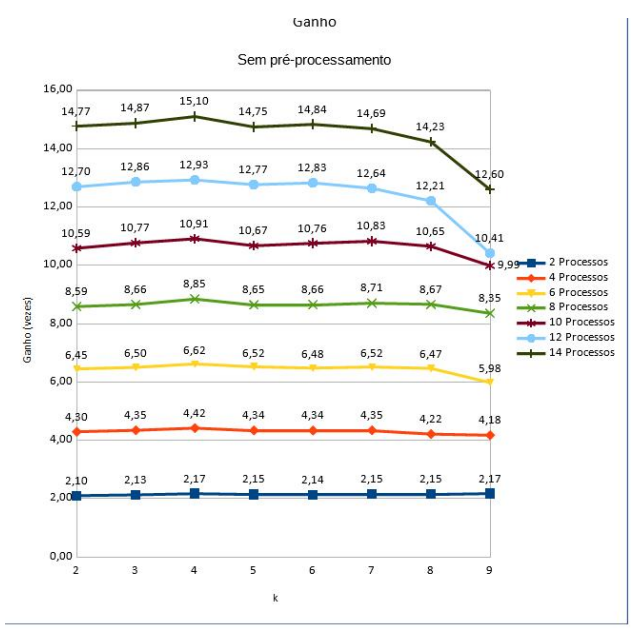

(a) Ganho do MCFRK sem o préprocessamento

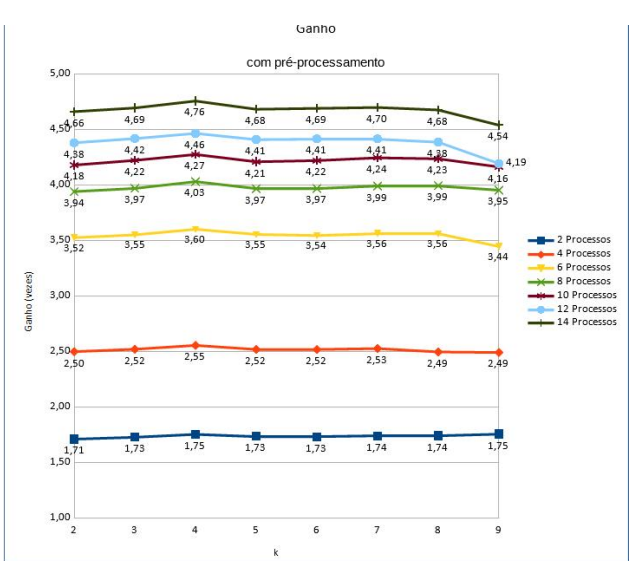

(b) Ganho do MCFRK com o préprocessamento

Figura 2: Ganho do MCFRK

de entrada em arquivos menores para serem enviados para os nós de processamento da cluster. Isto constata o gargalo existente nos algoritmos que realizam muitas operações de leitura e escrita em disco.

Ao compararmos as Figuras $2 \mathrm{a}$ e $2 \mathrm{~b}$ podemos observar que a medida em que aumentamos o número de processos, aumentamos o grau de execução em paralelo do algoritmo ao mesmo tempo em que diminuímos o tamanho do arquivo a ser processado em cada nó. Isto gera a diminuição no tempo de processamento por nó apresentada na figura 2a, porém o tempo de execução da operação split permanece o mesmo, aumentado a proporção do tempo de execução da operação split em relação as outras operações o que explica a diminuição do speed-up apresentada na figura $2 \mathrm{~b}$.

Demostramos que o CFRK, ao acrescentar o terceiro nível de paralelismo através da execução em uma cluster híbrida composta por nós de processamento com duas GPU's cada, é capaz de apresentar um ganho "supralinear"para a maioria dos valores de $k$. O mesmo experimento mostra que ao contabilizar os gastos com o pré-processamento, a otimização em três níveis diminui e passa para um ganho de até 3.84 vezes com 14 nós de processamento, conforme a Figura $2 \mathrm{~b}$. Observamos que a medida em que aumentamos os nós de processamento, aumentamos o grau de execução em paralelo do algoritmo ao mesmo tempo em que diminuímos o tamanho do arquivo a ser processado em cada nó, ou seja, diminuímos a taxa de "cache miss"gerando um ganho "supralinear"no algoritmo MCFRK, conforme a Figura 2a. Por outro lado o tempo de execução da operação split se manteve constante, aumentado a proporção do tempo de execução da operação split em relação as outras operações e causando a diminuição no ganho total, conforme a Figura $2 b$.

Por final, podemos observar que o ganho de desempenho obtido com as otimizações implementadas neste trabalho possibilitou o aumento na eficiência do algoritmo MCFRK que passou a apresentar bom desempenho para valores de $k$ até 9 , conforme podemos observar nas Figuras $2 \mathrm{a}$ e $2 \mathrm{~b}$. 


\section{Conclusão e trabalhos futuros}

Neste trabalho apresentamos otimizações desenvolvidas no algoritmo CFRK, originalmente desenvolvido para implementar paralelismo ao nível de threads em uma GPU, para permitir a execução com mais 3 níveis de paralelismo. Em um primeiro nível, utilizamos as diretivas do padrão OpenMP para permitir o processamento de módulos do algoritmo em múltiplos núcleos da $C P U$. Em um segundo nível, utilizamos as bibliotecas do modelo de programação pthread para permitir o processamento de mais de uma instância do módulo GPU em múltiplas GPU's. Por final, em um terceiro nível, utilizamos as bibliotecas do modelo de programação $M P I$ para permitir a execução de múltiplos processos do algoritmo CFRK em nós de processamento de uma cluster.

Nossos experimentos demonstram que a paralelização ao nível dos múltiplos núcleos da $C P U$, para um nó de 12 núcleos, gerou um ganho de 34 vezes em relação a execução com apenas um núcleo. Este ganho mostra que a paralelização no ambiente multicore, além de aumentar a taxa de execução em paralela do algoritmo, diminui o tempo de acesso aos dados na memória, gerando um ganho "supralinear"para a função SelectChunk. Demonstramos também que ao acrescentarmos o segundo nível de paralelismo através da execução do algoritmo CFRK em duas GPU's, o tempo de execução diminuiu de $39 \%$ ao invés de $50 \%$ devido a latência de transferência de dados entre a GPU e a $C P U$. Demostramos que o CFRK, ao acrescentar o terceiro nível de paralelismo através da execução em uma cluster híbrida composta por nós de processamento com duas GPU's cada, também apresenta um ganho "supralinear"para a maioria dos valores de $k$ devido ao aumento do grau de execução em paralelo do algoritmos e à diminuição do tamanho do arquivo a ser processado em cada nó e consequente diminuição da taxa de "cache miss". Por final, o experimento mostra que ao contabilizar os gastos com o pré-processamento, a otimização em três níveis tem um ganho muito menor, em até 4.78 vezes para 14 nós. Isto se dá porque apesar de aumentarmos o grau de execução em paralelo e diminuirmos a taxa de "cache miss", à medida em que aumentamos o número de nós de processamento, a latência da operação de transferência de dados se mantém constante, aumentando proporcionalmente o tempo de execução em transferência de dados em relação ao tempo de execução de processamento e causando uma diminuição no ganho total, conforme apresentado na Figura $2 b$.

Como trabalho futuro pretendemos desenvolver otimizações no CFRK de forma a diminuir o gargalo gerado pelas transferências de dados de entrada e saída. Pretendemos também avaliar o desempenho do CFRK em arquiteturas que apresentam um canal de alto desempenho para transferência de dados entre o host e a GPU tais como o nvlink da NVIDIA. Como trabalhos futuros também pretendemos acoplar o algoritmo CFRK

a ferramentas para a análise de amostras de material genético para ser utilizado pelos usuários do portal de bioinformática do LNCC.

\section{Referências}

Church, P. C., Goscinski, A., Holt, K., Inouye, M., Ghoting, A., Makarychev, K., and Reumann, M. (2011). Design of multiple sequence alignment algorithms on parallel, distributed memory supercomputers. In 2011 Annual International Conference of the IEEE Engineering in Medicine and Biology Society, pages 924-927. IEEE. 
Coelho, M., Vilasboas, F., and Osthoff, C. (2016). Desenvolvimento de uma versão paralela híbrida para a contabilização da frequência de repetição de k-mers. ERAD-RJ Escola Regional de Alto Desempenho do Rio de Janeiro.

Edwards, R. A., Olson, R., Disz, T., Pusch, G. D., Vonstein, V., Stevens, R., and Overbeek, R. (2012). Real time metagenomics: using k-mers to annotate metagenomes. Bioinformatics, 28(24):3316-3317.

Erbert, M., Rechner, S., and Müller-Hannemann, M. (2017). Gerbil: a fast and memoryefficient k-mer counter with gpu-support. Algorithms for Molecular Biology, 12(1):9.

Fiannaca, A., La Rosa, M., Rizzo, R., and Urso, A. (2015). A k-mer-based barcode DNA classification methodology based on spectral representation and a neural gas network. Artificial intelligence in medicine, 64(3):173-84.

Garg, A., Jain, A., and Paul, K. (2013). GGAKE: GPU Based Genome Assembly Using K-Mer Extension. In 2013 IEEE 10th International Conference on High Performance Computing and Communications \& 2013 IEEE International Conference on Embedded and Ubiquitous Computing, pages 1105-1112. IEEE.

Marçais, G. and Kingsford, C. (2011). A fast, lock-free approach for efficient parallel counting ofoccurrences of k-mers. Bioinformatics (Oxford, England), 27(6):764-70.

Plaza Onate, F., Batto, J.-M., Juste, C., Fadlallah, J., Fougeroux, C., Gouas, D., Pons, N., Kennedy, S., Levenez, F., Dore, J., Ehrlich, S. D., Gorochov, G., and Larsen, M. (2015). Quality control of microbiota metagenomics by k-mer analysis. BMC genomics, 16(1):183.

Vilasboas, F., Osthoff, C., Trelles, O., and Vasconcelos, A. T. (2015). Desenvolvimento de um algoritmo paralelo para contabilização da repetição de k-mers. $3^{a}$ Conferência Ibero Americana de Computação Aplicada 2015.

Vilasboas, F., Osthoff, C., Trelles, O., and Vasconcelos, A. T. (2016). Otimização de um algoritmo paralelo para contabilização da repetição de k-mers. II Escola Regional de Computação de Alto Desempenho do Rio de Janeiro. 